\title{
ON UNIVERSAL QUADRATIC NULL FORMS IN FIVE VARIABLES*
}

BY

\author{
R. S. UNDERWOOD
}

\section{INTRODUCTION}

1. We shall use L. E. Dickson's result: $†$

THEOREM 1. Every universal quadratic null form in three or more variables is equivalent to a form

$$
F=2^{e} g a x y+g b y^{2}+c y z+g d \psi(z, w, \cdots) \quad(e \geqq 0),
$$

where $g$ and a are odd, $a$ is prime to $d, c$ is prime to $g$, and the greatest common divisor of the coefficients of $\psi$ is 1 .

We investigate the case of five variables. In (1) let

$$
\psi=\alpha\left(h z^{2}+j z w+l w^{2}\right)+A z v+B w v+C v^{2},
$$

where

(3) 1 is the greatest common divisor of $\alpha, A, B, C$ and of $h, j, l$, and where, by an argument which carries over from Dickson's paper, $h$ may be taken prime to any given odd integer. We take $h$ prime to $g a$.

We shall assume that one of $N, M, P \neq 0$, where $N=j^{2}-4 h l, M=A^{2}-4 \alpha h C$, $P=2 h B-j A$. For if $N=M=P=0$, then $4 \alpha h \psi=(2 \alpha h z+\alpha j w+A v)^{2}$, where either $\alpha j w+A v$ is identically zero or it may be taken as a product of a constant by a new variable $w$. Hence this case reduces to the problem for three or four variables.

2. We shall need the following lemmas:

LEMMA 1. If each of the congruences

$$
F \equiv G \quad\left(\bmod 2^{e}\right), \quad F \equiv G \quad(\bmod g a y),
$$

with $F$ as in (1) and (2), has a solution $x, y, z, w, v$ such that $y$ is odd, then $F=G$ is solvable.

* Presented to the Society, June 13, 1931; received by the editors in December, 1930.

$\dagger$ Universal quadratic forms, these Transactions, vol. 31, No. 1, pp. 164-189. Subsequent references to the four-variable case refer to this paper. 


\section{LEMMA 2. The congruence}

$$
f=a x^{2}+b x y+c y^{2} \equiv k
$$

$\left(\bmod p^{n}\right)$,

where $p$ is an odd prime, is solvable for every $k$ if and only if $\Delta=b^{2}-4 a c \neq 0$ $(\bmod p)$ when $n=1$, and $(\Delta / p)=1$ when $n>1$.

Proof of Lemma 1. Since gay is odd, (4) implies $F \equiv G(\bmod P y)$, where $P=2^{\text {ega }}$. But $F=P x y+\phi, \phi \equiv G(\bmod P y), \phi=G+P y Q$. Hence $F=G$ when $x=-Q$.

Proof of Lemma 2. Case 1. $a \equiv 0, b \neq 0(p)$. Let $y=1$. Then $f \equiv b x+c \equiv k(p)$, which is solvable. Assume $f=k+H p^{m}, m \geqq 1$. Then $f \equiv k\left(p^{m+1}\right)$ is solvable with $x$ replaced by $x+X p^{m}$, since $H+b X \equiv 0(p)$ has a solution $X$. In this case $\Delta \equiv b^{2}(p)$, hence $(\Delta / p)=1$.

Case 2. $a \equiv b \equiv 0(p)$. Then $f \equiv c y^{2}(p)$ and $f \equiv k(p)$ is not solvable for some $k$. In this case $\Delta \equiv 0(p)$.

Case 3. $a \neq 0(p)$. Multiplying (5) by $4 a$ we get the equivalent congruence

$$
Z^{2}-\Delta y^{2} \equiv 4 a k\left(p^{n}\right)
$$

where

$$
Z=2 a x+b y .
$$

When $\Delta \equiv 0(p),(6)$, hence (5) also, is not solvable for some $k$. When $\Delta \not \equiv(p)$, it is well known that (6) is solvable modulo $p$. The solutions $Z, y$ fix $x$, modulo $p$, by (7), so that $x, y$ satisfy (5), modulo $p$.

Consider $n>1$. If $(\Delta / p)=-1, k=p K$ requires $y \equiv Z \equiv 0(p)$, whence (6) is not satisfied modulo $p$ for some $K$. If $(\Delta / p)=1$, the pair $Z, y$ which satisfy (6) modulo $p$ may be chosen so that one of $Z, y$, hence one of $x, y \neq 0(p)$. Let $j=k+H p^{m}, m \geqq 1$, one of $x, y \neq \equiv(p)$. Then $a\left(x+X p^{m}\right)^{2}+b\left(x+X p^{m}\right)\left(y+Y p^{m}\right)$ $+c\left(y+Y p^{m}\right)^{2} \equiv k\left(p^{m+1}\right)$ if

$$
H+M X+N Y \equiv 0(p),
$$

where $M=2 a x+b y, N=b x+2 c y$. Congruence (8) is satisfied unless $M \equiv N$ $\equiv 0(p)$, i.e., unless $\Delta x \equiv \Delta y \equiv 0(p)$, which contradicts the hypotheses on $\Delta, x$, and $y$.

$$
\text { The CONGRUence } F \equiv G(\bmod g a y)
$$

3. Preliminary results. Discussion of $y$. We may factor $g$ and $a$ as follows: (9) $g=q r, a=s t, q$ and $s$ have the same distinct prime factors; $r$ and $t$ are prime to each other and to both $q$ and $s$.

Then $q s, r$ and $t$ are relatively prime in pairs. Thus $F \equiv G$ is solvable 
modulo $g a$ if solvable modulis $q s, r$ and $t$. It is solvable modulis $q s$ and $r$ in the four-* and hence the five-variable case. It remains to consider

$$
F=G(t y) \text {. }
$$

Let $t$ be the product of powers $p^{n}$ of distinct primes. We shall use the following theorem:

THEOREM 2. If for each factor $p^{n}$ of $t=p^{n_{1}} \cdots p_{r}^{n_{r}}$, (11) $F \equiv G\left(p^{n}\right)$, with $F$ as in (1) and (2), has a solution $z$, w, v, $y=\eta$, with $\eta=1$ or $\pi$, where

(12) $\pi$ is an odd prime dividing no one of $g, a, d, \alpha, h$, and not dividing one of $N=j^{2}-4 h l, M=A^{2}-4 \alpha h C, P=2 h B-j A$, then (10) is solvable with $y$ odd but not necessarily the same as in the solution of (11).

Note that by the last paragraph of $\$ 1$

(13) one of $N, M, P \neq 0$.

First we prove

LEMMA 2A. The congruence

(14) $F \equiv G(\pi), \pi$ as in (12), is solvable with $y=k \pi$, where $k$ is an arbitrary integer.

For, dropping the terms of $F$ containing $y$, and multiplying (14) by $4 \alpha h$, we get the equivalent congruence

$$
4 \alpha h F \equiv g d\left(Z^{2}-\alpha^{2} N w^{2}-M v^{2}+2 \alpha P w v\right) \equiv 4 \alpha h G(\pi),
$$

where $Z=2 \alpha h z+\alpha j w+A v$, and $N, M, P$ are as in (12). Since $2 \alpha h$ is prime to $\pi$ we may take $Z$, $w, v$ as new variables in place of $z, w, v$. If $N=M=0$, then $P \neq 0$ by (13), and since $2 g d \alpha P$ is prime to $\pi$ by (12), (15) is solvable. Otherwise with $v=0$ or $w=0$ according as $N \neq 0$ or $N=0, M \neq 0,(15)$ is solvable by Lemma 2 , and hence, since $4 \alpha h$ is prime to $\pi$, (14) is solvable. This completes the proof of Lemma 2A.

Then by hypothesis (11) has a solution $z^{\prime}, w^{\prime}, v^{\prime}, y=\eta$, where $\eta=1$ or $\pi$, and by Lemma $2 \mathrm{~A}$, if $\eta=\pi$ (and trivially if $\eta=1$ ) $F \equiv G(\eta)$ has a solution $z^{\prime \prime}, w^{\prime \prime}, v^{\prime \prime}, y=\eta$.

By the Chinese Remainder Theorem there exist integers $z, w, v$ such that $z \equiv z^{\prime}, w \equiv w^{\prime}, v \equiv v^{\prime}\left(\bmod p^{n}\right)$, and $z \equiv z^{\prime \prime}, w \equiv w^{\prime \prime}, v \equiv v^{\prime \prime}(\bmod \eta)$. Then (11) and (14) have the same solution $z, w, v, y=\eta$, and hence, since $\eta$ is prime to $a$ and therefore to $p$,

$$
F \equiv G\left(p^{n} \eta\right)
$$

\footnotetext{
* With $v=w=0$, change $h$ to $\alpha h$ in line 9, p. 174 , of the reference previously quoted.
} 
has the same solution $z, w, v, y=\eta$.

For each factor $p^{n}$ of $t=p_{1}^{n_{1}} \cdots p_{r}^{n_{r}}$ there are then solutions $z_{i}, w_{i}, v_{i}$, $y=\eta_{i}$ of $F \equiv G\left(p_{i}^{n_{i}} \eta_{i}\right)$, where $\eta_{i}=1$ or a prime $\pi_{i}$ of type (12). Taking, by the Chinese Remainder Theorem,

$$
Y \equiv \eta_{i}, Z \equiv z_{i}, W \equiv w_{i}, V \equiv v_{i} \quad\left(\bmod p_{i}{ }^{n_{i}} \eta_{i}\right) \quad(i=1, \cdots, r),
$$

we have that $F \equiv G(t \zeta)$ has a solution $Y, Z, W, V$, where $\zeta=\eta_{1} \eta_{2} \cdots \eta_{r}$. Since by (17) $Y=\eta_{i}\left(1+k_{i} p_{i}^{n_{i}}\right)$, where $n_{i} \geqq 1, i=1, \cdots, r$, it follows that $Y=\zeta S$, where $S$ contains no factor $p$ of $t$. We select a prime $\tau=S+X_{1} t$ of type (12) and not dividing $t \zeta$ from the infinite number of the form $S+X t$, where $X$ ranges over all integers, and take $y=\zeta \tau$. Then $F \equiv G(t \zeta)$ has a solution $Z, W, V, y=\zeta \tau=\zeta S+X_{1} t \zeta$, and, by Lemma $2 \mathrm{~A}, F \equiv G(\tau)$ has a solution $Z^{\prime}, W^{\prime}, V^{\prime}, y=\zeta \tau$. Since $\tau$ is prime to $t \zeta$ it follows from the Chinese Remainder Theorem that $F \equiv G(t \zeta \tau)$ has a solution $Z^{\prime \prime}, W^{\prime \prime}, V^{\prime \prime}, y=\zeta \tau$. This completes the proof of Theorem 2.

In applying Theorem 2 we shall proceed in practice as follows. We seek a solution of (11) $F \equiv G\left(p^{n}\right)$, and find that various conditions on the coefficients in $F$ require the consideration of numerous sub-cases. As a first step we consider

$$
F \equiv G(p) .
$$

When Theorem 2 is applicable we can either set $y=1$ explicitly or show that there is a solution of (18) with $y=r \neq 0(p)$. From the infinite number of primes of the form $r+X p$, where $X$ runs through all integral values, we select a prime $\pi$ of type (12) and set $y=\pi$. Then where convenient we make the induction proving (11) solvable with $y$ fixed. When we make the induction through $y$ we show explicitly that $y$ remains prime to $p$, so that at each stage of the induction it can be chosen a prime of type (12) selected from the infinite number of primes of the form $\eta+X p^{m}, \eta \neq 0(p), m \geqq 1$.

There remain, besides the certainly non-universal cases, those for which we can prove (18) solvable but find it not convenient, and in some cases not possible, to hold $y \neq 0(p)$ in the solution. In such cases, as in 12123 below, we use the following theorem:

THEOREM 2A. Let $p^{n}$ be a typical factor of $t=p_{1}^{n_{1}} \cdots p_{r}^{n_{r}}$. If, for $F$ as in (1) and (2), $F \equiv G\left(p^{\sigma}\right)$ has for every $\sigma \geqq n$ a solution $z, w, v, y=p^{\delta} \eta$, where $\eta=1$ or a prime of type (12) and $\delta \geqq 0$ depends upon $G, p$, n, and $F$, but not upon $\sigma$, then (10) $F \equiv G(t y)$ is solvable.

Let $s=n+\delta$. By hypothesis $F \equiv G\left(p^{s}\right)$ has a solution $z, w, v, y=p^{\delta} \eta$, where $\eta=1$ or a prime of type (12). Also, by Lemma $2 \mathrm{~A}, F \equiv G(\eta)$ has a solution 
$z^{\prime}, w^{\prime}, v^{\prime}, y=p^{\delta} \eta$. Hence, applying the Chinese Remainder Theorem, $F \equiv G\left(p^{s} \eta=p^{n} y\right)$ has a solution $Z, W, V, y=p^{\delta} \eta$. That is, $F \equiv G\left(p_{i}^{n_{i}} y_{i}\right)$ has a solution $z_{i}, w_{i}, v_{i}, y_{i}=p_{i}{ }^{\delta_{i}} \eta_{i}$, where $i=1, \cdots, r$ and each of the $\eta_{i}$ is 1 or a prime of type (12).

From this point the part of the proof of Theorem 2 below (16) applies exactly if we delete the now unnecessary statement "where $\eta_{i}=1$ or a prime $\pi_{i}$ of type (12)" and elsewhere replace $\eta_{i}$ by $y_{i}$. Note that $\zeta=\eta_{1} \eta_{2} \cdots \eta_{r}$ becomes $\zeta=y_{1} y_{2} \cdots y_{r}$.

In view of Theorem $2 \mathrm{~A}$ it will suffice in practice to prove that, excluding the (here) provably non-universal cases, congruence (11) $F \equiv G\left(p^{n}\right)$ has a solution $z, w ; v, y=p^{\delta} r, r \neq 0(p)$, where $\delta$ is unchanged in the induction from modulus $p^{m}$ to modulus $p^{m+1}$. This will be true when $y$ remains fixed in the induction, and will be shown explicitly when the induction is through $y$.

4. The congruence $F \equiv G(\bmod t)$. Since $t$ is prime to $g, d$, and $h$, but divides $a$, the congruence

$$
F \equiv G\left(p^{n}\right),
$$

where $p$ is a prime factor of $t$, is equivalent to

$$
F=g b y^{2}+c y z+g d\left[\alpha\left(h z^{2}+j z w+l w^{2}\right)+A z v+B w v+C v^{2}\right] \equiv G\left(p^{n}\right) .
$$

We may assume without loss of generality that

$$
G \not \equiv 0\left(p^{2}\right) \text {. }
$$

For suppose $G=p^{2 s} G_{1}$, where $G_{1} \not \equiv 0\left(p^{2}\right)$. If $n \leqq 2 s,(20)$ is solvable with $y=p^{n}$, $z=w=v=0$. If $n>2 s$, take $y=p^{s} y^{\prime}, z=p^{s} z^{\prime}, w=p^{s} w^{\prime}, v=p^{s} v^{\prime}$. Division by $p^{2 s}$ yields the equivalent congruence $F\left(y^{\prime}, z^{\prime}, w^{\prime}, v^{\prime}\right) \equiv G_{1}\left(p^{n-2 s}\right)$, which justifies the assumption.

In the following scheme of subdivision, 121 and 122 are subheads of 12 , etc.

1. $C \neq 0(p)$.

11. $b \equiv c \equiv D \equiv E \equiv K \equiv 0(p)$,

where $D=4 \alpha h C-A^{2}, E=2 \alpha j C-A B, K=4 \alpha l C-B^{2}$. Then $F$ is not universal.

Multiplying both sides of (20) by $4 C$, we have

(22) $F_{1}=4 C F=g d\left[V^{2}+D z^{2}+2 E z w+K w^{2}\right]+4 C\left(g b y^{2}+c y z\right) \equiv 4 C G\left(p^{n}\right)$,

where $V=2 C v+A z+B w$. Since our moduli are powers of $p$ we may take $V, z, w, y$ as new variables in place of $v, z, w, y$, and (22) is equivalent to (20). By the conditions of $11, F_{1} \equiv g d V^{2} \equiv 4 C G(p)$, which is not solvable for some $G$.

12. One of $b, c, D, E, K \neq 0(p)$.

121. $b \neq 0(p)$. Вy (22) 


$$
F_{2}=g b F_{1}=C Y^{2}-L z^{2}+g^{2} d b\left[V^{2}+2 E z w+K w^{2}\right] \equiv k\left(p^{n}\right),
$$

where

$$
Y=2 g b y+c z, L=R C+g^{2} d b A^{2}, \quad R=c^{2}-4 g^{2} d b \alpha h, \quad k=4 g b C G .
$$

1211. $\left(-4 g^{2} d b C / p\right)=(-d b C / p)=1$. By Lemma 2, (23) is solvable with $z=w=0$.

1212. $(-d b C / p)=-1$.

12121. One of $L, E, K \neq 0(p)$. Then (23) is solvable.

Take $V=1$. Then (23) has a solution $Y, z, w$, modulo $p$, with (1) $w=0$, (2) $z=0$, (3) $z=1$ according as (1) $L \neq \equiv$, (2) $L \equiv 0, K \not \equiv 0,(3) L \equiv K \equiv 0$, $E \not \equiv 0(p)$. Assume $F_{2}=k+H p^{m}, m \geqq 1, V \not \equiv 0(p)$. Then $F_{2} \equiv k\left(p^{m+1}\right)$ with $Y, z, w$ (and hence $y$ ) unchanged and with $V$ replaced by $V+p^{m} X \not \equiv 0(p)$, since $2 g^{2} d b V \not \equiv 0(p)$.

12122. $L=p L_{1}, E=p E_{1}, K=p K_{1}, T=g^{2} d b E_{1}^{2}+L_{1} K_{1} \equiv 0(p)$. Then $F$ is not universal.

Since $(-d b C / p)=-1$, the solution of (23) modulo $p$ with $k=p k_{1}$ requires $Y \equiv V \equiv 0(p)$, so that, dividing out $p, F \equiv p k_{1}\left(p^{2}\right)$ reduces to $-L_{1} z^{2}$ $+g^{2} d b\left(2 E_{1} z w+K_{1} w^{2}\right) \equiv k_{1}(p)$, which by Lemma 2 is not solvable for some $k_{1}$.

12123. $L=p L_{1}, E=p E_{1}, K=p K_{1}, T \not \equiv 0(p)$. Then (23) is solvable.

By Lemma 2 we may fix $Y$ and $V$ modulo $p$ so that

$$
C Y^{2}+g^{2} d b V^{2} \equiv k(p),
$$

hence $k-C Y^{2}-g^{2} d b V^{2}=p Q$. Then (23) modulo $p^{2}$ reduces to

$$
-L_{1} z^{2}+g^{2} d b\left(2 E_{1} z w+K_{1} w^{2}\right) \equiv Q(p),
$$

which is solvable by Lemma 2 . This fixes $z$ and $w$, modulo $p$, and hence also $y$ and $v$ through $Y$ and $V$. Assume $F_{2}=k+H p^{m}, m \geqq 2$, and assume first $V \not \equiv 0(p)$. Then $F_{2} \equiv k\left(p^{m+1}\right)$ with $Y, z, w$ unchanged and with $v$ replaced by $v+p^{m v^{\prime}}$ (so that $V$ is replaced by $V+2 C p^{m} v^{\prime}$ ), since $2 g^{2} d b V \not \equiv 0(p)$.

It remains to complete the induction on (23) from $m \geqq 2$ to $m+1$ when $V \equiv 0(p)$. First assume $k \neq 0(p)$, so that $Y \not \equiv 0(p)$ by (24). If $y \not \equiv 0(p)$ we complete the induction by replacing $y$ by $y+p^{m} y^{\prime} \not \equiv 0(p)$, so that $Y=2 g b y+c z$ is replaced by $Y+2 g b p^{m} Y^{\prime} \not \equiv 0(p)$. If $y \equiv 0(p)$ then $c z \not \equiv 0(p)$. We set $y=p$ and replace $z$ by $z+p^{m} Z$, hence $Y$ by $Y+c p^{m} Y^{\prime} \not \equiv 0(p)$ and $V=2 C v+A z+B w$ by $V+A p^{m} Z \equiv 0(p)$. The induction is complete since $2\left(g^{2} d b A V+c C Y\right) \equiv 2 c C Y$ $\not \equiv 0(p)$. 
Next assume $k=p k_{1}$, where $k_{1} \not \equiv 0(p)$ by $(21)$. Then since $(-d b C / p)=-1$, $Y \equiv V \equiv 0(p)$ is required. Note that $c \equiv 0(p)$ then requires that $y \equiv 0(p)$. Since $k_{1} \neq 0(p), Q \neq 0(p)$ by the equation just below (24), hence one of $z, w \neq 0(p)$ in the solution of (25). Assume $F_{2}=k+H p^{m}, m \geqq 2$, one of $z, w \neq 0(p)$. Then $F_{2} \equiv k\left(p^{m+1}\right)$ with $z$ and $w$ replaced respectively by $z+p^{m-1} Z, w+p^{m-1} W$ if $C\left(Y+c p^{m-1} Z\right)^{2}+g^{2} d b\left(V+A p^{m-1} Z+B p^{m-1} W\right)^{2} \equiv C Y^{2}+g^{2} d b V^{2}\left(p^{m+1}\right)$ and $H+2 \theta_{1} Z+2 g^{2} d b \theta_{2} W \equiv 0(p)$, where $\theta_{1}=-L_{1} z+g^{2} d b E_{1} w, \theta_{2}=E_{1} z+K_{1} w$. The latter congruence is solvable, since $\theta_{1} \equiv \theta_{2} \equiv 0(p)$ requires that $T z \equiv T w \equiv 0(p)$, contradicting the conditions on $T, z$, and $w$. The former congruence is also solvable if $Y \equiv V \equiv 0\left(p^{2}\right), m \geqq 3$. For the case $m=2$, with $z$ and $w$ fixed by (25) we hold $Y \equiv V \equiv 0\left(p^{2}\right)$ by adjustment of $y$ and $v$. The latter need not be changed thereafter, since if $Y \equiv V \equiv 0\left(p^{2}\right)$ then $Y+p^{m-1} c Z \equiv V+p^{m-1}(A Z$ $+B W) \equiv 0\left(p^{2}\right)$ for $m \geqq 3$.

122. $b \equiv 0, c \neq 0(p)$. By Lemma $2,(20)$ is solvable with $w=v=0$, since the $\Delta$ of Lemma $2=c^{2}-4 g^{2} d b h \equiv c^{2}(p)$, hence $(\Delta / p)=1$.

123. $b=p b_{1}, c=p c_{1}, D \not \equiv 0(p)$. By (22)

$$
F_{3}=g d D F_{1}=g^{2} d^{2} D V^{2}+Z^{2}+g^{2} d^{2} \theta w^{2}+4 p \delta_{1} C y^{2}-4 p \gamma_{1} y w \equiv k\left(p^{n}\right),
$$

where

$$
\begin{aligned}
& Z=g d D z+g d E w+2 c C y, \theta=D K-E^{2}, \quad \delta=g^{2} b d D-c^{2} C=p \delta_{1}, \\
& \gamma=g d c C E=p \gamma_{1}, \quad k=4 g d C D G .
\end{aligned}
$$

Applying to (26) the arguments of 121 as applied to (23), we find (26) solvable unless $\theta=p \theta_{1},(-D / p)=-1$, and $\gamma_{1}{ }^{2}-g^{2} d^{2} C \theta_{1} \delta_{1} \equiv 0(p)$, in which case $F$ is not universal. To make sure that the power of $p$ in $y$ is not indefinitely increased in the induction we note that by the argument of 121 as obviously modified to fit the new lettering the induction is through $V$ and $Z$ (i.e., $v$ and $z$ ) unless $k=p k_{1}, k_{1} \neq 0(p), V \equiv Z \equiv 0(p)$, with one of $y, w \equiv 0(p)$. Then if $y \equiv 0(p), \theta_{1} w \neq 0(p)$, and the induction can be made through $w$ alone; otherwise $y$ remains $\not \equiv 0(p)$ in the required replacement of $y$ by $y+p^{m-1} Y$.

124. $b=p b_{1}, c=p c_{1}, D=p D_{1}, K \not \equiv 0(p)$. By (22)

$$
F_{4}=K F_{1}=g d\left[W^{2}+K V^{2}+\theta z^{2}\right]+4 p C K\left(g b_{1} y^{2}+c_{1} y z\right) \equiv 4 C K G\left(p^{n}\right),
$$

where

$$
W=K w+E z, \theta=D K-E^{2} .
$$

As in 123, (27) is solvable unless $\theta=p \theta_{1},(-K / p)=-1$, and $c_{1}{ }^{2} C K-g^{2} d b_{1} \theta_{1}$ $\bar{\equiv} 0(p)$, in which case $F$ is not universal. 
125. $b=p b_{1}, c=p c_{1}, D=p D_{1}, K=p K_{1}, E \not \equiv 0(p)$.

In this case (22) is solvable by Lemma 2 with $V=0, y=p^{n} \eta$, since the $\Delta$ of Lemma $2 \equiv 4 E^{2}(p)$, hence $(\Delta / p)=1$.

2. $C \equiv 0(p)$, and one of $A, B \not \equiv(p)$. Then (20) is solvable.

Fix $z$ and $w$ so that $A z+B w=M \not \equiv 0(p)$, and take $y=1$. Then (20) yields (28)

$$
L(v)=g d\left(M v+C v^{2}\right) \equiv k\left(p^{n}\right),
$$

where $k$ is a constant. This is solvable, modulo $p$. Assume $L(v)=k+H p^{m}$, $m \geqq 1$, and take $v^{\prime}=v+X p^{m}$. Then

$$
\begin{aligned}
L\left(v^{\prime}\right) & \equiv k+p^{m}[H+g d M X]\left(p^{m+1}\right) \\
& \equiv k\left(p^{m+1}\right)
\end{aligned}
$$

by choice of $X$, completing the induction.

3. $A=p A_{1}, B=p B_{1}, C=p C_{1}$. Then $\alpha \neq \equiv(p)$ by (3).

31. $N=j^{2}-4 h l \not \equiv 0(p)$.

Since $p$ is odd and does not divide $g d \alpha h$, multiplication of (20) by $4 g d \alpha h$ yields the equivalent congruence

(29) $4 g d \alpha h\left(g b y^{2}+c y z\right)+g^{2} d^{2} \alpha^{2}\left[(2 h z+j w)^{2}-N w^{2}\right]+\xi \equiv 4 g d \alpha h G\left(p^{n}\right)$, where

$$
\xi=4 p g^{2} d^{2} \alpha h\left(A_{1} z+B_{1} w+C_{1} v\right) v .
$$

The product of (29) by $N$ gives

(31) $S(U, V, y, z, w, v)=N U^{2}-V^{2}+J y^{2}+4 p D\left(A_{1} z v+B_{1} w v+C_{1} v^{2}\right) \equiv k\left(p^{n}\right)$, where

(32) $U=g d \alpha(2 h z+j w)+c y$,

(33) $V=N g d \alpha w+c j y$,

(34) $J=c^{2} j^{2}-N R, R=c^{2}-4 g^{2} d b \alpha h, k=4 g d \alpha h N G, D=N g^{2} d^{2} \alpha h \not \equiv 0(p)$.

311. $J \not \equiv 0(p)$.

312. $(N / p)=1$.

In cases 311 and $312,(20)$ is solvable with $v=0$, as shown in the fourvariable case.

313. $(N / p)=-1$ and $J=p J_{1}$. 
The result is given in

(35) $\{$ when $Q \equiv 0(p), F$ is not universal;

when $Q \not \equiv 0(p),(31)$ is solvable, where $Q=\alpha C_{1} J_{1} N-c^{2} h\left(j B_{1}-2 A_{1} l\right)^{2}$.

Since $N \not \equiv 0(p)$,

(36) one of $j, l \neq \equiv 0(p)$.

Since $J \equiv 0(p)$, (31) gives

$$
N U^{2}-V^{2} \equiv k(p) .
$$

This is solvable by Lemma 2, fixing $U$ and $V$ modulo $p$. It remains to test (31) modulo $p^{m}, m \geqq 2$.

3131. $C \equiv 0(p)^{2}$.

31311. $c \equiv 0(p)$. Then $F$ is not universal.

For, by (37), (33), and (32), $k=p K$ requires $U \equiv V \equiv w \equiv z \equiv 0(p)$. By (31), $S \equiv J y^{2}\left(p^{2}\right) \not \equiv p K\left(p^{2}\right)$ for some $K$.

31312. $\mu=2 A_{1} l-j B_{1} \equiv 0(p)$. Then $F$ is not universal.

Eliminating $y$ from (32) and (33) and replacing $j^{2}-N$ by $4 h l$, we get

$$
j U-V \equiv 2 g d \alpha h(j z+2 l w)(p) .
$$

Multiplying (38) by $B_{1}$ and replacing $j B_{1}$ by $2 A_{1} l$, we get

$$
(j U-V) B_{1} \equiv 4 g d \alpha h l M(p),
$$

where

$$
M=A_{1} z+B_{1} w .
$$

By (37) and (39) $k=p K$ requires $U \equiv V \equiv l M \equiv 0(p)$. The condition $l \equiv 0(p)$ requires $j \neq 0(p)$ by (36), whence by (38) and the condition that $\mu \equiv 0(p), z \equiv B_{1} \equiv 0(p)$. Hence $k=p K$ requires $M \equiv 0(p)$. By (31) $S \equiv J y^{2}\left(p^{2}\right)$ $\not \equiv p K\left(p^{2}\right)$ for some $K$.

31313. $c \mu \neq 0(p)$. Then (31) is solvable.

Since $\mu=2 A_{1} l-j B_{1} \not \equiv 0(p)$,

$$
j B_{1}=2 A_{1} l+r \text {, where } r \not \equiv 0(p) .
$$

Multiplying (38) by $B_{1}$ and replacing $j B_{1}$ by $2 A_{1} l+r$, we get

$$
(j U-V) B_{1}-2 g d \alpha h r z \equiv 4 g d \alpha h l M\left(p^{n}\right) \text {, with } M \text { as in (40). }
$$

Noting (36) and the fact that $U$ and $V$ are fixed, modulo $p$, by (37), we have three subcases: 
When $l \equiv 0(p), j B_{1} \equiv r \not \equiv(p)$ by (41), and $z$ is fixed modulo $p$ by (38). Choose $w$ so that, by (40), $M \neq \equiv(p)$. Then $y$ is fixed modulo $p$ by (32) or (33) (consistent through (38)).

When $j \equiv 0(p), 2 A_{1} l \equiv-r \not \equiv 0(p)$ by (41), and $w$ is fixed modulo $p$ by (33). Choose $z$ so that, by (40), $M \neq \equiv(p)$, fixing $y$ modulo $p$ by (32).

When $j l \neq 0(p)$, choose $z$ so that the left side of $(42) \not \equiv 0(p)$.

In all cases we have $M=A_{1} z+B_{1} w \neq 0(p)$. By (31), taking $C=p^{2} C_{2}$,

$$
L(v)=4 D p\left(M v+p C_{2} v^{2}\right) \equiv K\left(p^{n}\right),
$$

where

$$
K=k-N U^{2}+V^{2}-J y^{2}=p K_{1},
$$

with $K_{1}$ independent of $v$. Dividing out $p$ we find (43)., hence (31), solvable by the method used for (28).

Note that (35) is satisfied in our results for 3131 .

3132. $C=p C_{1}, C_{1} \neq \equiv(p)$.

31321. $c \equiv J_{1} \equiv 0(p)$. Then $F$ is not universal.

By (37), (33) and (32), $k=p K$ requires $U \equiv V \equiv w \equiv z \equiv 0(p)$. By (31), noting $J=p J_{1}, S \equiv 4 p D C_{1} v^{2}\left(p^{2}\right) \not \equiv p K\left(p^{2}\right)$ for some $K$.

31322. $c \equiv 0, J_{1} \neq 0(p)$. Then (31) is solvable.

$U, V, w$ and $z$ are fixed, modulo $p$, by (37), (33), and (32). Multiplying both sides of (31) by $C_{1}$, we get

$$
p C_{1} J_{1} y^{2}+p D \Delta^{2} \equiv K\left(p^{2}\right),
$$

where

$$
\begin{aligned}
& \Delta=2 C_{1} v+A_{1} z+B_{1} w, \\
& K=C_{1}\left(k-N U^{2}+V^{2}\right)+p D\left(A_{1} z+B_{1} w\right)^{2}=p K_{1} .
\end{aligned}
$$

Dividing out $p$, we find (44) solvable by Lemma 2 , fixing $y, \Delta$, and $v$, modulo $p$, by (44) and (45). Thus (31) is solvable modulo $p^{2}$. The induction will be completed in 313234 .

31323. $c \not \equiv 0(p)$.

313231. $j \equiv P \equiv 0(p), P=\alpha C_{1} J_{1}+c^{2} l A_{1}^{2}$. Then $F$ is not universal.

By (32) we have, with $j \equiv 0(p)$,

$$
c^{2} y^{2} \equiv(U-2 g d \alpha h z)^{2}(p) .
$$

Multiplying (44) by $c^{2}$, replacing $K$ and $c^{2} y^{2}$ by their values in (46) and (47) respectively, and then $D$ in the coefficient of $z$ by its value in (34), and noting that $N=j^{2}-4 h l \equiv-4 h l(p)$, we have 


$$
p D c^{2} \Delta^{2}+4 p \alpha h^{2} g^{2} d^{2} P z^{2}-p T z \equiv K_{2}\left(p^{2}\right)
$$

where

$$
\begin{gathered}
T=4 C_{1} J_{1} g d \alpha h U+2 c^{2} D A_{1} B_{1} w, \\
K_{2}=c^{2} C_{1}\left(k-N U^{2}+V^{2}\right)+p\left(D c^{2} B_{1}^{2} w^{2}-C_{1} J_{1} U^{2}\right)=p K_{3} .
\end{gathered}
$$

By (37), (33), and (49), $k=p K$ requires $U \equiv V \equiv w \equiv T \equiv 0(p)$, whence by (50) $K_{2} \equiv p c^{2} C_{1} K\left(p^{2}\right)$. We then have by (48) and the fact that $P \equiv 0(p)$

$$
p D c^{2} \Delta^{2} \equiv p c^{2} C_{1} K\left(p^{2}\right)
$$

which is not solvable for some $K$.

Note that $j \equiv P \equiv 0(p)$ is equivalent to $Q \equiv 0(p)$, with $Q$ as in (35).

313232. $j \equiv 0, P \not \equiv 0(p)$. Then (31) is solvable.

$U, V$, and $T$ are fixed, modulo $p$, by (37), (33), and (49). Completing the square in $z$ in (48) we have

$$
p \delta \Delta^{2}+p Z^{2} \equiv K_{4}\left(p^{2}\right)
$$

where

$$
\begin{aligned}
Z & =8 \alpha h^{2} g^{2} d^{2} P z-T, \\
K_{4} & =16 \alpha h^{2} g^{2} d^{2} P K_{2}+p T^{2}=p K_{5}, \delta=16 \alpha h^{2} g^{2} d^{2} P D c^{2} \not \equiv 0(p) .
\end{aligned}
$$

Dividing out $p$ we find (51) solvable by Lemma 2 , fixing $\Delta$ and $Z$, modulo $p$, and hence also $z, v$, and $y$ by (52), (45), and (32) respectively. Thus (31) is solvable modulo $p^{2}$. The induction will be completed in 313234 .

313233. $j \not \equiv 0, Q \equiv 0(p)$, with $Q$ as in (35). $F$ is not universal.

Eliminating $y$ and $z$ from (44) (with $K$ replaced as in (46)) by means of (33) and (38), we get

$$
p\left(E \Delta^{2}+L w^{2}-M w\right) \equiv K_{6}\left(p^{2}\right),
$$

where

$$
\begin{gathered}
E=D(2 c j g d \alpha h)^{2} \not \equiv 0(p), \\
L=D h(2 g d \alpha)^{2} Q, \\
M=4 g d \alpha h D\left[2 \alpha C_{1} J_{1} V+c^{2} A_{1}(j U-V)\left(j B_{1}-2 A_{1} l\right)\right], \\
K_{6}=(2 g d \alpha h c j)^{2} C_{1}\left(k-N U^{2}+V^{2}\right) \\
\\
\quad+p\left[c^{2} D A_{1}^{2}(j U-V)^{2}-C_{1} J_{1}(2 g d \alpha h V)^{2}\right]=p K_{7} .
\end{gathered}
$$

By (37) and (57), $k=p K$ requires $U \equiv V \equiv M \equiv 0(p)$. Since $Q$ and hence $L \equiv 0(p)$, we get, from (54) and (58), $p E \Delta^{2} \equiv p(2 g d \alpha h c j){ }^{2} C_{1} K\left(p^{2}\right)$, which is not solvable for some $K$. 
313234. $j Q \not \equiv 0(p)$, with $Q$ as in (35). Then (31) is solvable.

$U, V$, and $M$ are fixed, modulo $p$, by (37) and (57). Completing the square in (54), we get

$$
4 p L E \Delta^{2}+p W^{2} \equiv K_{8}\left(p^{2}\right)
$$

where

$$
\begin{aligned}
& W=2 L w-M, \\
& K_{8}=4 L K_{6}+p M^{2}=p K_{9} .
\end{aligned}
$$

Dividing out $p$ we find (59) solvable by Lemma 2 , fixing $\Delta$ and $W$, modulo $p$, and hence also $w, y, z$ and $v$ by (60), (33), (32) and (45) respectively. Thus (31) is solvable modulo $p^{2}$.

It remains to complete the induction in cases 31322,313232 , and 313234 . This will be done in subdivisions of 313234 . For convenience we may rewrite (31) as follows:

$$
S=N U^{2}-V^{2}+p J_{1} y^{2}+4 p D Z v+4 p D C_{1} v^{2} \equiv k\left(p^{n}\right),
$$

where

$$
Z=A_{1} z+B_{1} w, \quad D g d \alpha h N C_{1} \not \equiv 0(p)
$$

and $y, z, w, v$ are fixed, modulo $p$. Since (62) is solvable modulo $p^{2}$ with the exceptions noted, we may have $m \geqq 2$ in the induction.

3132341. $U \neq \equiv 0(p)$.

Assume $S(z)=k+H p^{m}$. Take $z^{\prime}=z+X p^{m}$. Then, by (32), $U^{\prime}=U+$ $2 h g d \alpha X p^{m} \neq \equiv 0(p)$. By (62) $S\left(z^{\prime}\right) \equiv k+p^{m}[H+4 g d \alpha h N X]\left(p^{m+1}\right)$, etc., completing the induction.

3132342. $U \equiv 0, V \not \equiv 0(p)$.

Assume $S(w)=k+H p^{m}$. Take $w^{\prime}=w+X p^{m}$. Then by (32) and (33), $U^{\prime}=U+g d \alpha j X p^{m} \equiv 0(p), \quad V^{\prime}=V+g d \alpha N X p^{m} \not \equiv 0(p)$. By $\quad(62) \quad S\left(w^{\prime}\right) \equiv k$ $+p^{m}\left[H-2 g d \alpha N V^{\prime} X\right]\left(p^{m+1}\right)$, etc., completing the induction.

3132343. $U \equiv V \equiv 0(p)$.

31323431. $v \not \equiv 0(p)$.

Assume $S(y, z, w, v)=k+H p^{m}$. Take $z^{\prime}=z+X p^{m-1}$. Then by (32) and the definition of $Z$ below (62), $U^{\prime}=U+2 h g d \alpha X p^{m-1} \equiv 0(p), Z^{\prime}=Z+A_{1} p^{m-1}$, and $S\left(z^{\prime}\right) \equiv k+p^{m-1}\left[H p+4 L_{1} X\right]\left(p^{m+1}\right)$, where $L_{1}=N U h g d \alpha+p D A_{1} v$. The induction will be complete unless $L_{1} \equiv 0\left(p^{2}\right)$. Similarly, taking in succession 
$y^{\prime}=y+X p^{m-1}$ (except when $\left.y \equiv 0(p)\right)$ and $w^{\prime}=w+X p^{m-1}$, we get in succession the two following coefficients of $X$ :

$$
\begin{aligned}
& L_{2}=2\left(N c U-c j V+p J_{1} y\right), \\
& L_{3}=2\left(g d \alpha j N U-g d \alpha N V+2 p D B_{1} v\right) .
\end{aligned}
$$

Hence with the proper substitution the induction is complete unless $y \equiv 0(p)$ or

$$
L_{1} \equiv L_{2} \equiv L_{3} \equiv 0(p) .
$$

Eliminating $U$ and $V$ from (63) and dividing out $p$, we get

$$
2 c D v\left(2 A_{1} l-j B_{1}\right)+N g d \alpha J_{1} y \equiv 0(p) .
$$

This will be considered in conjunction with (67) below.

Again, take $z^{\prime}=z\left(1+X p^{m-1}\right), y^{\prime}=y\left(1+X p^{m-1}\right), w^{\prime}=w\left(1+X p^{m-1}\right)$. Then since $U \equiv V \equiv 0(p)$, we have $U^{\prime}=U\left(1+X p^{m-1}\right) \equiv 0(p), V^{\prime}=V\left(1+X p^{m-1}\right)$ $\equiv 0(p), Z^{\prime}=Z\left(1+X p^{m-1}\right)$. Finally, take $v^{\prime}=v\left(1+X p^{m-1}\right) \not \equiv 0(p)$. The induction will be valid in one of these last two cases unless

$$
L_{4} \equiv L_{5} \equiv 0(p)
$$

where

$$
L_{4}=2\left(J_{1} y^{2}+2 D Z v\right), L_{5}=4 D v\left(Z+2 C_{1} v\right) ;
$$

$Z$ is as defined below (62). Eliminating $Z$ from (65), we get

$$
4 C_{1} D v^{2}-J_{1} y^{2} \equiv 0(p) .
$$

Since $4 C_{1} D v \neq 0(p),(67)$ is impossible if $y \equiv 0(p)$; hence in that case one of $L_{4}, L_{5} \neq 0(p)$, and the induction is complete with the power of $p$ in $y$ unaltered.

Finally, eliminating $y$ from (64) and (67), replacing $D$ by $N g^{2} d^{2} \alpha h$, and dropping common factors which we know to be prime to $p$ (as $g, d, \alpha, h, N, C$ ), we get $v^{2} Q \equiv 0(p)$, with $Q$ as in (35), which contradicts the hypotheses on $Q$ and $v$. Hence at least one of the five induction substitutions above succeeds, and the induction is complete.

31323432. $v \equiv 0(p)$.

By (21) we may assume that $G \not \equiv 0\left(p^{2}\right)$, hence in (62) $k=4 g d \alpha h G N \not \equiv 0\left(p^{2}\right)$. Then by (62) $J_{1} y \neq 0(p)$. Assume $S(y, z, w, v)=k+H p^{m}$, where $U \equiv V \equiv 0$, $J_{1} y \neq 0(p)$. Take $y^{\prime}=y\left(1+X p^{m-1}\right) \not \equiv 0(p), z^{\prime}=z\left(1+X p^{m-1}\right), w^{\prime}=w\left(1+X p^{m-1}\right)$. Then $U^{\prime}=U\left(1+X p^{m-1}\right) \equiv 0(p), V^{\prime}=V\left(1+X p^{m-1}\right) \equiv 0(p), Z^{\prime}=Z\left(1+X p^{m-1}\right)$. 
By (62) $S\left(y^{\prime}, z^{\prime}, w^{\prime}, v^{\prime}\right) \equiv k+p^{m}\left[H+2 J_{1} y^{2} X\right]\left(p^{m+1}\right)$, etc., completing the induction. This completes 313. Examination of the subcases reveals that (35) is a complete statement of the results.

32. $N=p N_{1}$. Then, since $N \equiv 0, h \neq 0(p)$,

$$
\text { either } j \equiv l \equiv 0 \text {, or } j l \neq 0(p) \text {. }
$$

321. $j l \neq 0(p)$. Then (31) is solvable, as shown in the four-variable case $(\$ 14, \mathrm{I})$.

322. $j \equiv l \equiv 0(p)$.

By (29),

$$
\begin{aligned}
\phi & =Z^{2}-R y^{2}+g^{2} d^{2} \alpha\left[\alpha j\left(4 h z w+j w w^{2}\right)-\alpha p N_{1} w^{2}+4 p h\left(A_{1} z v+B_{1} w v+C_{1} v^{2}\right)\right] \\
& \equiv k\left(p^{n}\right),
\end{aligned}
$$

where

$$
\begin{aligned}
& Z=2 g d \alpha h z+c y, \\
& R=c^{2}-4 g^{2} d \alpha h b .
\end{aligned}
$$

By (69) and 322 we have

$$
Z^{2}-R y^{2} \equiv k(p)
$$

3221. $(R / p)=1$. Then by Lemma $2,(69)$, hence (19), is solvable with $w=v=0$.

3222. $R \equiv 0(p)$. Then $F$ is not universal.

For then (72) has no solution $Z$ for some $k$.

3223. $(R / p)=-1$. The result is as follows:

$$
\left\{\begin{array}{l}
\text { when } I \equiv 0(p), F \text { is not universal; } \\
\text { when } I \not \equiv 0(p),(69) \text { is solvable, where } I=h B_{1}{ }^{2}+\alpha N_{1} C_{1} .
\end{array}\right.
$$

$Z, y$ and $z$ are fixed, modulo $p$, by (72) and (70). It remains to determine when (69) is solvable modulis $p^{2}$ and $p^{n}$.

32231. $C_{1} \equiv B_{1} \equiv 0(p)$. Then $F$ is not universal.

For, by (72) and (70), $k=p K$ requires $Z \equiv y \equiv z \equiv 0(p)$. By (69), $\phi \equiv$ $-p g^{2} d^{2} \alpha^{2} N_{1} w^{2}\left(p^{2}\right) \not \equiv p K\left(p^{2}\right)$ for some $K$.

32232. $C_{1} \equiv 0, B_{1} \neq 0(p)$. Then (69) is solvable.

Choose $w$ so that $A_{1} z+B_{1} w=M \not \equiv(p)$. By (69)

$$
4 g^{2} d^{2} \alpha h p M v \equiv K\left(p^{2}\right) .
$$


where

$$
\left.K=k-Z^{2}+R y^{2}-g^{2} d^{2} \alpha^{2}\left(4 h j z w+j^{2} w^{2}\right)=p K_{1} \text { (independent of } v\right) .
$$

Dividing out $p$ we find (74) solvable. Using (69), assume $\phi(v)=\phi(y, z, w, v)$ $=k+p^{m} H$, with $m \geqq 2$. Take $v^{\prime}=v+X p^{m-1}$. Then $\phi\left(v^{\prime}\right) \equiv k+p^{m}[H$ $\left.+4 g^{2} d^{2} \alpha h M X\right]\left(p^{m+1}\right)$, etc., completing the induction.

32233. $C_{1} \neq \equiv 0, I \equiv 0(p)$, with $I$ as in (73). $F$ is not universal.

Completing the square in (69), we get

$$
p E V^{2}+\delta w^{2}+\epsilon w \equiv K_{1}\left(p^{n}\right),
$$

where

By (72) and (70), $k=p K$ requires $Z \equiv y \equiv z \equiv 0(p)$, and hence, since $j \equiv I \equiv 0(p), \delta \equiv \epsilon \equiv 0\left(p^{2}\right)$. Then by (75) and (80),

$$
p E V^{2} \equiv p C_{1} K\left(p^{2}\right)
$$

which is not solvable for some $K$.

32234. $C_{1} I \not \equiv 0(p)$, with $I$ as in (73). Then (69) is solvable.

$Z, y$ and $z$ are fixed, modulo $p$, by (72) and (70). Writing $\delta=p \delta^{\prime}, \epsilon=p \epsilon^{\prime}$ (since $j \equiv 0(p)$ ), we have by (75) and (80), after dividing out $p$,

$$
E V^{2}+\delta^{\prime} w^{2}+\epsilon^{\prime} w \equiv K_{2}\left(p^{n-1}\right),
$$

where $\delta^{\prime} \equiv-g^{2} d^{2} \alpha I \not \equiv 0(p)$. Since $E \delta^{\prime} \not \equiv 0(p)$, we may complete the square in $w$ and have (82) solvable modulo $p$, fixing $V, w$ and $v$, modulo $p$, by (82) and (76). Hence (69) is solvable modulo $p^{2}$.

Using (69), assume $\phi=k+p^{m} H$, with $m \geqq 2$. By (70), if $Z \equiv 0(p)$, either $y \neq 0(p)$ or $z \equiv y \equiv 0(p)$. Also, if $Z \equiv z \equiv y \equiv 0(p)$ we may assume that one of $N_{1} w, v \neq 0(p)$, since $j \equiv 0(p)$ and by $(21) k$ may be taken $\not \equiv 0\left(p^{2}\right)$. The following induction substitutions then cover all cases:

(a) $Z \not \equiv 0(p)$. Take $z^{\prime}=z+X p^{m}$, hence $Z^{\prime}=Z+2 g d \alpha h X p^{m} \not \equiv 0(p)$.

(b) $Z \equiv 0, \quad y \neq 0(p)$. Take $z^{\prime}=z\left(1+X p^{m}\right), \quad y^{\prime}=y\left(1+X p^{m}\right)$, hence $Z^{\prime}$ $=Z\left(1+X p^{m}\right) \equiv 0(p)$. 
(c) $Z \equiv z \equiv y \equiv B_{1} w \equiv 0, v \not \equiv 0(p)$. Take $v^{\prime}=v+X p^{m-1}$.

(d) $Z \equiv z \equiv y \equiv N_{1} \equiv 0, B_{1} w v \not \equiv 0(p)$. Take $w^{\prime}=w+X p^{m-1}$.

(e) $Z \equiv z \equiv y \equiv B_{1} v \equiv 0, N_{1} w \neq \equiv(p)$. Take $w^{\prime}=w+X p^{m-1}$.

(f) $Z \equiv z \equiv y \equiv 0, B_{1} N_{1} w v \not \equiv(p)$. Take $w^{\prime}=w+X p^{m-1}, v^{\prime}=v+Y p^{m-1}$. Then $\phi \equiv k+p^{m}[H+L X]\left(p^{m+1}\right)$, etc., completing the induction in cases (a) to (e) inclusive, where $L \neq \equiv(p)$ and is as follows:

(a) $4 g d \alpha h Z$; (b) $-2 R y^{2}$; (c) $8 g^{2} d^{2} \alpha h C_{1} v$; (d) $4 g^{2} d^{2} \alpha h B_{1} v$; (e) $-2 g^{2} d^{2} \alpha N_{1} w$. In case $(\mathrm{f}), \phi \equiv k\left(p^{m+1}\right)$ unless

$$
S \equiv T \equiv 0(p)
$$

where

$$
S=2 h B_{1} v-\alpha N_{1} w, T=2 C_{1} v+B_{1} w .
$$

But on eliminating $w,(83)$ is equivalent to $2 v I \equiv 0(p)$, contradicting the hypotheses, hence (83) is impossible and the induction is complete.

The results in the subcases of $\mathbf{3 2 2 3}$ satisfy (73).

We have now proved the solvability of (19), and hence, by Theorems 2 and $2 \mathrm{~A}$, of (10), except in cases (84) below.

Let $p$ be any prime dividing $a$ but not $g$. Let the appearance of $b_{1}$ indicate that $b=p b_{1}$, of $D_{1}$ that $D=p D_{1}$, etc. Define

$D=4 \alpha h C-A^{2}, \quad E=2 \alpha j C-A B, \quad K=4 \alpha C C-B^{2}, \quad R=c^{2}-4 g^{2} d b \alpha h$,

$L=R C+g^{2} d b A^{2}, \theta=D K-E^{2}, \quad \delta=g^{2} d b D-c^{2} C, \gamma=g d c C E$,

$N=j^{2}-4 h l, \quad J=c^{2} j^{2}-N R, \quad I=h B_{1}-\alpha N_{1} C_{1}$,

$Q=\alpha C_{1} J_{1} N-c^{2} h\left(j B_{1}-2 l A_{1}\right)^{2}$.

Then $F$ is not universal in the following cases:

1. $C \not \equiv 0(p)$, and one of 11 to 14 holds:

11. $b \equiv c \equiv D \equiv E \equiv K \equiv 0(p)$;

12. $(-d b C / p)=-1, g^{2} d b E_{1}^{2}+L_{1} K_{1} \equiv 0(p)$;

13. $(-D / p)=-1, \gamma_{1}^{2}-g^{2} d^{2} C \theta_{1} \delta_{1} \equiv 0(p)$;

14. $(-K / p)=-1, c_{1}^{2} C K-g^{2} d_{1} b \theta_{1} \equiv 0(p)$;

2. $A \equiv B \equiv C \equiv 0(p)$, and one of 21,22 holds:

21. $J \equiv Q \equiv 0(p),(N / p)=-1$;

22. $N \equiv j \equiv 0(p)$, and either $R \equiv 0(p)$ or $(R / p)=-1$ and $I \equiv 0(p)$.

Since $y$ is odd we then have, by Lemma 1 ,

Theorem 3. If $e=0, F$ is universal except in cases (84). 


\section{REMARKS ON THE CONGRUENCE $F \equiv G\left(\bmod 2^{n}\right)$}

5. The briefer results. The initial problem was solved completely in the writer's thesis, but the conditions upon the coefficients as found are so numerous that the bare listing of the results, except those given below for the case $e=1$, requires a prohibitive amount of space. We shall omit further proofs and close with the statements of two fundamental lemmas which give additional freedom in the choice of $y$, and of the theorem for the case $e=1$.

Lемма 3. If there exists an odd y satisfying $(4)_{2}$, it may be chosen congruent to any desired odd residue, modulo $2^{n}$.

LEMMA 4. If the solution of

$$
F \equiv G\left(2^{e}\right)
$$

requires the factor $2 \cdot$ in $y$, where $s \geqq 0$ depends upon $G$, then $F$ is universal, subject to exceptions (84), if and only if

$$
F \equiv G\left(2^{e+s}\right)
$$

is solvable with $x=0$ for every pair $G, s$.

ThEOREM 4. When $e=1, F$ is universal subject to (84) unless (a), bcdah is odd, $l \equiv B \equiv C \equiv 0(4), j$ and $A$ are even, or (b), $d=2 D, c$ is even, and $b$ or $D$ is even. If either (a) or (b) holds, $F$ is not universal.

University of Chicago,

Chicago, Ill. 